\title{
Brecha digital, categorías perceptivas y cognitivas: El caso de los video-juegos
}

\author{
Rafael DEL VILLAR MUÑOZ*
}

\section{Introducción}

La brecha digital no es simplemente un desigual acceso a la cultura en el mundo postmoderno, sino que ella se inserta en una problemática más trascendente vinculada a la emergencia de una sociedad en redes que implica operar con habilidades cognitivas y perceptivas ligadas al uso de las nuevas tecnologías que, de no tenerlas, imposibilita el acceso al empleo por no tenerse las competencias requeridas. En ese contexto, la investigación semiótica que aquí sintetizamos se torna relevante, pues aborda el tema de la descripción de las categorías perceptivas y cognitivas presentes en los video-juegos más consumidos en Chile: Estrategia, Aventura Gráfica y Combate, de consumo diferencial según estrato social.

Los video-juegos son un objeto de estudio estratégico desde el punto de vista de la descripción semiótica de las categorías cognitivas y perceptivas de las nuevas generaciones, pues ellos son, por una parte, un objeto de alto consumo infante-juvenil $\mathrm{y}$, por otra parte, el consumo infante-juvenil es fundamentalmente multimedial, implicando una forma de funcionamiento hipertextual y multi-soporte.

En ese contexto, el video-juego como objeto real a intelegibilizar se torna relevante, pues "el video-juego mismo es multimedial en su funcionamiento", implica personajes, los personajes remiten virtualmente a historias, historias que no están en el texto sino que fuera de él, desarrollan un tratamiento visual (cromatismo, estructuración lineal, etc.) ligado a un tipo de diseño gráfico que se expresa en revistas, sitios web, filmes, libros, etc.; "por otra parte, el video-juego es interactivo y presupone un consumidor participativo" que construye sus propias vías de juego, a partir de una matriz central que da la máquina, estableciéndose una interactividad similar a la vida cotidiana.

Luego, los video-juegos son susceptibles de cristalizar fractalmente, en el sentido de la geometría fractal de la naturaleza ${ }^{1}$ los procesos cognitivos y perceptivos implicados, de este segmento infante juvenil. La geometría fractal de la naturaleza es próxima al "paradigma holográfico de los físicos cuánticos", ellos nos dicen que en un sub-conjunto de lo real, analizado en profundidad, ampliado mil veces metafóricamente permite dar las bases para construir el todo: "La parte está en el todo y el todo está en cada parte, una especie de unidad-en-la-diversidad y diversidad-en-la-unidad. El punto crucial es sencillamente que la parte tiene acceso al todo" ${ }^{2}$. Mandelbrot nos plantea que si antes la geometría buscaba la regularidad, las invariantes de las formas de la realidad, la geometría fractal de la naturaleza propuesta por él busca en lo irregular, en lo fragmentado los principios constitutivos de una totalidad. "La matemática clásica está enraizada en las estructuras regulares de la geometría de Euclides y en evolución continua de la dinámica de

\footnotetext{
* Académico e investigador del Instituto de la Comunicación e Imagen. Universidad de Chile.

${ }^{1}$ MANDELBROT, B."La geometría fractal de la naturaleza”. Editorial Tusquets. Barcelona, 1997.

${ }^{2}$ WILBER, K.; BOHM, D.; PRIBRAM,K., KEEN, S.,FERGUSON, M., CAPRA, F. Y OTROS. "El paradigma holográfico”. Pág. 8. Editorial Cairos. Barcelona, 1991.
} 
Newton.... la revolución se produjo al descubrirse estructuras matemáticas que no encajaban en los patrones de Euclides y Newton. Estas nuevas estructuras fueron consideradas patológicas" ${ }^{\text {. }}$. El número de irregularidades de la naturaleza fueron consideradas residuales, como mero mundo fenoménico. Pero el punto es que "el número de escalas de longitud de las distintas formas naturales es, a efectos prácticos, infinito", luego, desde el punto de vista de la formalización es imposible sólo ver regularidades, sino que cambiando el paradigma científico, podemos de la parte del fragmento construir el todo. El fragmento es una parte, pero al mismo tiempo es una irregularidad, no es la regla; pero de dicho fragmento es posible construir un principio constitutivo que puede presentarse en diferentes escalas. Así, Mandelbrot no busca la regularidad, lo que se repite en todos los árboles para entender un árbol desde el punto de vista geométrico, lo que hace es tomar un fragmento del árbol: una hoja, y ampliarlo mil veces, y en ese fragmento ampliado de la hoja, en los principios constitutivos de su nervadura están los principios geométricos que constituyen la naturaleza del árbol mismo. Es lo mismo que pretendemos hacer con nuestro modelo interpretativo de la forma de funcionamiento más profunda, de las categorías perceptivas e intelectivas, de estos procesos de construcción de identidades a lo que los video-juego, y, más general, la video-animación está ligada en función de caracterizar polos, tipos de funcionamiento. Hay, evidentemente, siguiendo a Mandelbrot un salto de escala desde estadísticas del consumo de medios de las nuevas generaciones, desde estadísticas de los video- juegos más gustados, a los procesos de funcionamiento de los protocolos perceptivos e intelectivos de los video-juegos mismos, y el puente entre esas escalas son los protocoles interpretativos perceptivos e intelectivos de los niños y jóvenes empíricos, cuyos modelos interpretativos puestos en actos iluminan, como fragmentos, construyendo una dimensión fractal que en su origen es el principio constitutivo de las distintas manifestaciones (en diferentes escalas de manifestación) de la experiencia real de nuestro objeto de estudio.

Debe tenerse en cuenta, además, que no sólo los video-juegos son consumidos por el segmento infante juvenil, sino que existe en la sociedad chilena un alto equipamiento de consolas para jugar video-juego. Estadísticas del Consejo Nacional de Televisión ${ }^{5}$ señalan que el equipamiento de video-juegos el año 2002 en el hogar es de 35,1\% en Antofagasta (Norte de Chile, zona minera), de un $17,5 \%$ en Temuco (Sur de Chile, zona forestal y ganadera) y de un $25,7 \%$ en Santiago (capital de Chile, zona industrial y de servicios), lo que es necesario interpretar en el contexto general de la estadística que es respecto a equipamiento de medios de la sociedad chilena, lo que hace elevar la importancia de la cifra, pues no todas las familias tienen niños y jóvenes que usen este tipo de tecnología (los muy niños, la tercera edad, ni los adultos jóvenes tienden a no consumir, en general, este tipo de objeto cultural).

Ahora bien, delimitado el lugar estratégico del video-juego como objeto real de estudio semiótico de las categorías cognitivas y perceptivas de las nuevas generaciones, y habiendo diferentes tipos de video- juego, compete definir cuales son los que permiten detectar nuestros objetivos de investigación. Diego Levis ${ }^{6}$ los clasifica en: 1) Juegos de lucha, 2) Juegos de Combate, 3) Juegos de Tiro, 4) Juegos de plataforma, 5) Simuladores,

\footnotetext{
${ }^{3}$ MANDELBROT, B. Op. Cit. Pág. 18.

${ }^{4}$ MANDELBROT, B. Op. Cit. Pág. 15.

${ }^{5}$ CONSEJO NACIONAL DE TELEVISIÓN. "Informe 8/3 Tweens Chilenos”. Pág. 49. Santiago, 2003.

${ }^{6}$ LEVIS, D. “Los video- juegos, un fenómeno de masas”. Pág. 168. Editorial Paidós. Barcelona, 1997.
} 
6) Deportes, 7) Juegos de Estrategia, 8) Ludo-educativos, 10) Porno-eróticos. Sin embargo, su clasificación dice referencia al contenido temático de los juegos, lo que es obviamente una vía, pero un poco lejos de nuestra perspectiva teórica, y de los delineamientos de la investigación que se sintetiza. Patricia M. Greenfield y Jean Retschitzki ${ }^{7}$ los clasifican "tomando como criterio de clasificación el tipo de la actividad que implican, distinguiendo cuatro grandes clases de juegos: juegos de acción, juegos de aventura, juegos de simulación y juegos de reflexión". Nosotros hemos tomado una vía empírica, y tomamos como base la investigación desarrollada por nuestro equipo de investigación en el Proyecto Fondecyt $\mathrm{N}^{\mathrm{o}}$ $1000954^{8}$, allí preguntamos a los sujetos mismos cuáles eran sus video-juegos más preferidos, y preguntamos las razones de gusto asociadas a dichos juegos preferidos, según estrato social, segmento etario y género. Los datos empíricos (300 encuestas estratificadas por estrato, segmento etario, género y ciudad) nos permitieron diagnosticar que los videojuegos más gustados son los de estrategia, aventura gráfica y combate, y su gusto es diferencial según estrato y género. Concretamente, en Santiago los video-juegos de combate gustan en un 38,5\% en estrato bajo, en un 38,5\% en medio-bajo, en medio-alto un $23,3 \%$, y en el alto un 22,6\%, esto es, los video-juegos de combate son los más gustados en los estratos medio-bajo y bajo; los video-juegos de estrategia gustan en el estrato bajo un $7,7 \%$, en el medio bajo un $46,2 \%$, en el medio alto un $56,7 \%$, y en el alto un $25,8 \%$, esto es, los video juegos de estrategia gustan más en el estrato medio alto y medio bajo, menos en el alto, y muy poco en el bajo; los video-juegos de aventura gráfica gustan en el estrato bajo en un $50 \%$, en el medio bajo un $46,5 \%$, en el medio alto en un $33,3 \%$, y en el alto un $29 \%$. Esto es, los video-juegos de aventura gráfica son más gustados en el estrato bajo, y medio bajo. Respecto al género, las mujeres prefieren aventura gráfica $(38,5 \%)$ por sobre combate y estrategia. En Antofagasta, aventura gráfica gusta en un $43 \%$ en el estrato alto, en el medio un 27\%, y en el bajo un 21\%; Combate en el estrato alto gusta en un $30 \%$, en el estrato medio un 43\%, y en el estrato bajo en un 33\%; estrategia gusta en el estrato alto en un $23 \%$, en el medio un 20\%, y en el bajo un 20\%; esto es en Antofagasta en el estrato medio y bajo prefieren combate, y en el alto aventura gráfica. En Temuco, en el estrato alto lo que más gusta es los video-juegos de aventura gráfica en un 33\%, y los de estrategia con un $30 \%$, los de combate solamente gustan en un $13 \%$, en el estrato medio los video-juegos de estrategia son los favoritos con un $30 \%$, y los de aventura gráfica en un $20 \%$, y los de combate un $25 \%$, en el estrato bajo prefieren los video-juegos de aventura gráfica y de estrategia, ambos con un $35 \%$ y el de combate con un $30 \%$; esto es, la preferencia por los video-juegos de combate es mucho más en el estrato socioeconómico bajo y medio, prácticamente el doble que en el estrato alto; los video-juegos de estrategia tienden a ser preferido por un tercio de los encuestados en cada estrato, lo que demuestra una tendencia bastante homogénea más allá del nivel socioeconómico; en los video-juegos de aventura gráfica las preferencias también ser acercan a un tercio de los encuestados, tanto en el estrato bajo como en el alto, mientras que en el medio esta preferencia cubre sólo a menos de un cuarto de los encuestados.

Luego, los datos empíricos avalan que el análisis semiótico debe anclar en describir los tres tipos de video-juegos más consumidos, como forma fractal del cual es posible detectar las categorías cognitivas y perceptivas implicadas.

\footnotetext{
${ }^{7}$ GREENFIELD, P. RETSCHITZKI, J.“L'enfant et les médias”. Pág. 142. Editorial Universitaires Fribourg Suisse. 1998.

${ }^{8}$ DEL VILLAR, R.;PERILLÁN L.; CASAS. "Proyecto Fondecyt No 1000954”, Santiago.
} 
Desde la perspectiva de la descripción de las categorías perceptivas y cognitivas de las nuevas generaciones, es claro que los datos empíricos nos detectan que hay un gusto y consumo diferencial por estrato social, y lo que llama la atención es la preferencia mayoritaria del gusto por los video-juegos de combate en el estrato bajo y medio bajo en Santiago, y en bajo y medio en Temuco, y, finalmente, lo mismo, en Antofagasta. También, es importante señalar, que por género, el consumidor femenino no gusta mucho de los video-juegos de combate.

Es claro, desde el punto de vista semiótico, por investigaciones anteriores sobre la forma de funcionamiento textual de los video-juegos ${ }^{9}$ que los video-juegos de combate son simples desde su perspectiva de operar con pocas variables de análisis (se trata de combatir, pegar o disparar a un contrincante), y pocos espacios a percibir (generalmente el espacio de combate), que los video-juegos de estrategia implican varios espacios perceptivos, y una multiplicidad de variables, que más que narrativas implican temáticas y sub-temáticas a solucionar, las que abren una multiplicidad de vías analíticas de operar en el juego, y que los video-juegos de aventura gráfica, si bien son eminentemente narrativos, la puesta en acto de un relato, implican a su vez, varios espacios perceptivos y estrategias disímiles.

Al mismo tiempo, nuestra investigación Proyecto Fondecyt $\mathrm{N}^{\mathrm{o}} 1030561^{10}$ detecta que las formas de jugar video-juegos no son formas específicas de asumir un juego concreto o un formato concreto, sino que las mismas categorías que se emplean para jugar son las que se aplican para procesar la vida cotidiana ${ }^{11}$. En esa investigación (estudio semiótico de 99 casos, estratificados por estrato, segmento etario, ciudad, y tipo de videojuego) se les pidió hacer un dibujo (segmento etario 5-6 años) o un guión (segmentos etarios 11-12 y 16-19 años), se les hizo jugar un video-juego, se grabó su forma de jugar, y al mismo tiempo una cámara registraba su comportamiento corporal y perceptivo), y se descubrió que el análisis semiótico de los dibujos o guiones coincidía con el análisis de su forma de jugar y con el registro gestual del jugador, y a través de una entrevista en profundidad realizada con posterioridad, se validó ${ }^{12}$ que las estructuraciones perceptivos y cognitivas que se daban en los tres registros, también se expresaban en la forma de enfrentar la vida cotidiana.

De allí, entonces, la relevancia del estudio semiótico de las categorías perceptivas y cognitivas presentes en los video-juegos de combate, estrategia y aventura gráfica, pues ello nos permitirá entender las formas de pensamiento intelectivo y perceptivo, y al mismo tiempo, describir el significado de los estudios cuantitativos de consumo y gusto de los video-juegos de combate, de aventura gráfica y estrategia, desde el punto de vista de detectar si dicho consumo retroalimenta o los aleja de una forma de funcionamiento emergente en las nuevas tecnologías de la producción que implican manejar habilidades cognitivas y perceptivas complejas, las que a simple vista parecen no estar implicadas en el consumo de video-juego de combate, hecho más preocupante, todavía en términos del porcentaje de gusto muy elevado en los segmentos socioeconómicos bajos.

\footnotetext{
${ }^{9}$ DEL VILLAR, R. "Videoculturas de fin de milenio: globalización, japoanimación y resemantización local", en "Revista Opción, año 17, N³6”. Ed. Universidad del Zulia. Maracaibo, 2001.

${ }^{10}$ DEL VILLAR, R.; CASAS, R.; PERILLÁN, L. "Proyecto Fondecyt No 1030561”. Santiago, 2004.

${ }^{11}$ DEL VILLAR, R. "Consommation de la vidéo- animation japonaise: redéfinition de la notion sémiotique et psychanalyse du sujet”, en Revue Visio, Vol. 8; No 2. Ed. Universidad de Laval. Québec, 2004.

12 DEL VILLAR, R. "Nuevas tecnologías y construcción de identidades", Revista de Signis No 5".Ed. Gedisa. Barcelona, 2004.
} 


\section{Semiótica cognitivista: categorías cognitivas y perceptivas}

Lo que las ciencias cognitivas descubren que el sujeto no capta al objeto real como relación estímulo-respuesta, el objeto real existe para el sujeto que a través de una sinápsis que lo aprehende a partir de sus propios principios de auto-organización: el fenómeno de la percepción como fenómeno de captación de rasgos de entes independientes, no puede ocurrir porque no hay mecanismo a través del cual tales entes pudiesen determinar lo que sucede en un sistema sensorial en una interacción. Esto es, el medio solo puede gatillar una percepción, pero ella es aprehendida a través de un sistema, y de sus propias reglas, no habiendo ni sensores ni efectores, como mecanismo estímulo-respuesta. No existe un sujeto que actúa, que conoce, que opera. Francisco Varela señala: "las estructuras cognitivas familiares de la vida humana emergen de esquemas recurrentes quienes permiten a la acción ser guiadas por la percepción"13. La hipótesis cognitivista no tiene viabilidad analítica: "en los cerebros reales no hay reglas, ni un operador lógico central, ni la información está almacenada en lugares precisos"14.

Por otra parte, uno de los vacíos o deficiencias de la hipótesis cognitivista es que el procesamiento de la información simbólica lógica se basa en reglas secuenciales aplicadas una por vez, luego es incapaz de comprender el procesamiento paralelo de la información, donde "los símbolos, en sentido convencional, no desempeñan ningún papel""

Lo anterior hace improcedente mucho desarrollo de las teorías de las comunicaciones y validan la perspectiva de $\mathrm{Jost}^{16}$ de crítica a aquellas teorías que presuponen, como P. Charaudeau ${ }^{17}$ que existe un contrato enunciativo entre emisores y receptores, lo que significa decir que bastaría el análisis de los contenidos presupuestos por los enunciadores (la video-animación), y el nivel de recepción de lo enunciado (el ratting de la video-animación) para saber las razones y las implicancias de su consumo, lo que no es más que un kantismo sin sujeto trascendental que reedifica la realidad.

Siguiendo a Jean Petitot-Cocorda ${ }^{18}$, desde un punto de vista cognitivo y semiótico, podemos situar, a nivel descriptivo, dos instancias o niveles de construcción perceptiva: -la noción de figura como contornos visibles, lo que correspondería a la noción de figura de la Gestall, y que J. Petitot-Cocorda define como: "partes desligables, en el sentido de pedazos"19, pedazos que podríamos traducir como fragmentos; y la noción de "partes no desligables o momentos como contornos, colores, entre otros, quienes siendo componentes necesarios de los objetos, implican con su extensión una relación de dependencia"20. Esto significa que son fragmentos asociativos que se desligan del objeto representando otra cosa, por relación asociativa en otro espacio o escala perceptiva.

Esto es, la percepción puede ir en correspondencia con estructuraciones simples de lo visible o compleja, es decir, es necesario distinguir dos tipos de segmentaciones de lo figural o experiencia sensible: los segmentos con contornos y figuras claras. Esto es, lo que

\footnotetext{
${ }^{13}$ VARELA, F. “Quel savoir pour l’éthique?. Pág. 29. Ed. La Découverte. París, 2004.

${ }^{14}$ VARELA, F. "Conocer". Pág. 53. Ed. Gedisa

${ }^{15}$ VARELA, F. Op. Cit. Pág. 77.

${ }^{16}$ JOST, F. “Introduction à l'Analyse de la Télévision”. Ed. Ellipsses. París, 1999.

17 CHARAUDEAU, P. "El discurso de la información. La construcción del espejo social". Ed. Gedisa. Barcelona, 2003.

${ }^{18}$ PETITOT-COCORDA, J. "Physique du sens”. Ed. CNRS. París, 2000.

${ }^{19}$ PETITOT-COCORDA, J. Op. Cit. Pág. 75.

${ }^{20}$ PETITOT-COCORDA, J. Op. Cit, Pág. 75.
} 
denominaríamos lo "real ingenuo" y la figuración conectiva de espacios perceptivos detectados más allá de las formas visibles (lo que Petitot- Cocorda ejemplifica como percepción de colores con colores, le líneas con líneas, más allá de su formato específico).

Respecto a las categorías perceptivas e intelectivas la descripción de PetitotCocorda, Varela, Pachoud, Roy ${ }^{21}$, nos permite tener un instrumental cognitivista actual respecto a la conceptualización de los protocolos que es necesario describir en la inteligibilización de nuestro objeto real. Desde esa perspectiva podemos detectar cuatro tipos o niveles de intelección y percepción de los objetos reales:

1) Relaciones de conjuntos, esto es, se percibe una relación simple a través de detectar la relación que opera con una parte de lo percibido: se detecta una variable; por ejemplo, el sujeto presiona el joistick y se mueve un personaje.

2) Relaciones de contigüedad; esto es, el sujeto realiza una operación de detectar que dos variables pueden estar conectadas por coexistir una cerca de la otra. No son un campo asociativo en el sentido semiótico del término, señala Petitot- Cocorda ${ }^{22}$, pues no se trata de correlacionar dos operaciones de sentido, sino que de una coexistencia de dos operaciones que van juntas, como por ejemplo, presionar el joistick, saltar, dar un golpe, bajar. Así, se detectan sub-cojuntos que pueden ir juntos;

3) Relaciones de conexión y dependencia, esto es, se detecta que dos operaciones pueden ligarse y producir otra, es la dependencia y la conexión de variables lo que se detecta; por ejemplo, un jugador puede elevarse, después dar un golpe, y posteriormente bajar,

4) Captación de momentos figurales que reagrupan los elementos en configuración, lo que significa tener un concepto de totalidad, y eventualmente construir una estrategia.

Luego, el saber acumulado de las ciencias cognitivas y de la semiótica cognitivista en la actualidad nos permiten tener los protocolos analíticos para la descripción del procesamiento perceptivo y cognitivo de los jugadores de video-juegos de combate, estrategia y aventura gráfica (se estudiaron 99 casos diferenciados por estrato social, segmento etario 5-6, 11-12, 16-19, y ciudad), teniendo claro tanto teóricamente como empíricamente que dicho procesamiento no dice relación sólo con los video-juegos, sino que con la vida cotidiana. De allí, la importancia de su estudio, pues nos permite reconstituir como fractal, un principio recurrente en diferentes escalas.

\section{Brecha digital $y$ estructuraciones cognitivas y perceptivas recurrentes en los usuarios de video-juego de combate (11-12 y 16-19 años)}

El video juego de combate es, desde el punto de vista de su funcionamiento textual, simple, pero se escogió para la investigación el video-juego de combate Dragón Ball, quien no deja de tener dificultades para los usuarios, pues tiene dos espacios (el espacio de la pelea y el espacio de una barra de herramientas que les dice el nivel de energía de vida y de poder, tanto de quién pelea como de su contrincante), lo que implica una mayor dificultad que los juego de combate comunes que sólo se circunscriben a la pelea. Esto significa que son posibles dos lecturas en el juego: una basada en el combate donde las habilidades cognitivas son simples, trabajar a un espacio o una lectura compleja donde se trabaja un procesamiento paralelo de la información. La lectura simple la pueden hacer todos, pero la

\footnotetext{
${ }^{21}$ PETITOT, J.; VARELA, F.;PACHOUD, B.;ROY, J.M. "Naturaliser la phénoménologie”. Ed. CNRS. París, 2002.

${ }^{22}$ PETITOT- COCORDA, J. Op.Cit.
} 
lectura de trabajar la información paralela de dos espacios y articularlas la hacen todos en Antofagasta, más de la mitad en Santiago y sólo un tercio en Temuco; lo que puede estar ligado al equipamiento de video-juego que en esta última ciudad es menor.

La cantidad de sujetos que hace una lectura simple del juego es preocupante desde el punto de vista productivo: un 37,5\% en Santiago y un 67\% en Temuco, significa decir que un porcentaje importante de sujetos no son competentes para un procesamiento paralelo de la información. Esta incapacidad cognitiva tiene enorme importancia en el desarrollo de actividades laborales que implican en el contexto de la actividad productiva más actual y tecnologizada, tener un procesamiento paralelo que establezca conexión y dependencia entre las tareas y procesamiento perceptivo paralelo.

Sin embargo, se detecta, además, una segunda categoría perceptiva importante con implicancias productivas: los video-jugadores de combate sólo perciben personajes, focalizando allí la mirada, independientemente de que algunos perciban dos espacios.

Luego, perceptivamente una recurrencia encontrada en los jugadores de juegos de combate es centrar la atención en el espacio narrativo de los personajes, lo que implica una forma de mirar que hemos encontrado recurrente en las tres ciudades y en todos los segmentos etéreos y de estrato social: por una parte en Temuco se privilegian los recorridos de miradas rectas, basados en una mirada horizontal izquierda-derecha y viceversa; y una mirada vertical arriba-abajo en línea recta (salvo un sujeto del estrato alto que miraba en forma recta diagonal), en Antofagasta encontramos el mismo dispositivo de recorrido de mirada, donde las líneas verticales y oblicuas eventuales permiten ligar el espacio barra de herramientas con el de la pelea; en Santiago en cambio esto no ocurre; la mitad de los sujetos manifestaron un movimiento de los ojos y de la cabeza similar a Antofagasta y Temuco, la otra mitad incorporó movimientos circulares de izquierda, margen inferior hacia arriba y después vuelta a margen inferior y viceversa. Sin embargo, estos recorridos no fueron predominantes en los casos que los insertaron. Otra característica de los recorridos de mirada detectados en los usuarios de video-juego de combate es la recurrencia de formas del mirar describibles bajo el patrón de horizontal-vertical y movimiento oblicuo en línea recta. Esto es no se trata de movimientos complejos (hechos de una multiplicidad azarística) de un recorrido que explore la totalidad, sino que ellos están condicionados por la focalización narrativa, se sitúan en el centro, por lo tanto, estamos ante la presencia de formas de mirar que son susceptibles de abstraer y transformarlas en figuras geométricas repetibles, correspondientes a la recurrencia de la geometría euclidiana, esto es, no son interpretables por la geometría fractal de la naturaleza. La tendencia entonces es a repetir ciertos patrones geométricos de recorrido que se caracterizan por ser pocos y además por ser líneas rectas, verticales, horizontales y oblicuas.

Luego, la percepción focaliza personajes y con ello se acostumbra a establecer recorridos de mirada regulares que corresponde a la pregunta narrativa (el combate) de un espacio, sin percibir ni articular recorridos de mirada que le permita percibir espacios paralelos. El mirar, entonces es el presupuestado para ejecutar una tarea productiva simple, por ejemplo, dar una respuesta a una pregunta concreta (estímulo respuesta) sin asociar ni percibir lo que pasa alrededor ni tampoco estar capacitado para responder ante tareas complejas que impliquen relacionar diferentes sub-conjuntos. 


\section{Brecha digital $\mathbf{y}$ estructuraciones cognitivas y perceptivas recurrentes en los usuarios de video-juego de estrategia (11-12 y 16-19 años)}

A los usuarios investigados se les hizo jugar Age of Empires II, quien permite a los jugadores seleccionar una de varias opciones para dominar el mundo y obtener la victoria: dominación de las civilizaciones enemigas (por la vía militar), victoria económica a través de la acumulación de la riqueza. (por la vía de los recursos), construyendo y defendiendo las maravillas del mundo (por la vía de la construcción de edificios). Age of Empires II permite a los jugadores escoger de entre numerosos caminos tecnológicos para ayudar a liderar una civilización desde la temprana edad media hasta la edad imperial, ya sea privilegiando estrategias económicas a través de la agricultura y la mano de obra o estrategias militares de bloqueo y ofensiva naval y terrestre. El juego viene con un "árbol de la tecnología" con más de 100 nudos. Luego, la estructuración cognitiva es compleja, pues remite a múltiples posibilidades de percepción espacial, dependiendo de las vías que implemente el consumidor. Los espacios básicos a partir del cual se construyen los múltiples son: la percepción de la pantalla; el minimapa, que ubica espacialmente el área en la cual se suceden los acontecimientos y las instrucciones lingüísticas. La estructura del juego no presupone la narratividad ni la linealidad, puesto que ésta la construye el consumidor.

El juego implicó una dificultad cognitiva, pues tanto en Temuco como en Santiago tiende la mitad a captarlo totalmente y la otra no; lo que implica que no todos los sujetos tienen la capacidad cognitiva de percibirlo. Algunos lo percibieron como video-juego de combate y otros como aventura gráfica independientemente de la ciudad de adscripción. Lo significativo es que esa dualidad no existe en Antofagasta.

Una característica del procesamiento cognitivo de los usuarios de video-juego de estrategia es la presencia de las siguientes categorías cognitivas relaciones de conjunto, contigüidad, conexión y dependencia y captación totalizadora de momentos figurales que permiten reagrupar los elementos en una configuración total, tanto en los usuarios mayoritarios de Santiago, Antofagasta y Temuco.

Otra característica cognitiva es que no se focalizan tan solo los personajes como objetos a percibir, sino que todos los espacios son susceptibles de percibirse y adquirir una significación. Esto aparece tanto en Santiago, Antofagasta y Temuco.

La recurrencia cognitiva encontrada en los usuarios de video-juegos de estrategia es que prima el articular de espacios paralelos y desarrollar estructuras perceptivas complejas. Las excepciones son propias de usuarios que no corresponden directamente al objetivo de describir el polo fractal de estrategia. Así, hay un sujeto que en Antofagasta (estrato bajo, 12 años) cree que es un juego de combate y trata de disparar lo que no tiene realidad en el juego, lo mismo ocurre en dos casos de estrato alto y medio bajo en Santiago y de dos casos en Temuco. El articular entonces espacios paralelos permite no sólo percibir espacios, sino que poder relacionarlos e implementar varias lógicas cognitivas operando a la vez. Recordemos que el procesamiento paralelo de la información no era entendido por las corrientes cognitivistas, pues para ellos la percepción era un conjunto de reglas lógicas que procesaba la información. Esta forma de funcionamiento cognitiva es entendida por el conexionismo, pues en la cognición operan sistemas conectivos de interrelaciones múltiples. No se trata entonces de "el procesamiento de la información simbólica basada en 
reglas secuenciales aplicadas una por vez... el cognitivismo simbólico es incapaz de comprobar un procesamiento paralelo de la información, pues lo localiza en secuencias" ${ }^{\text {23 }}$.

No existe una recurrencia de los jugadores de video-juegos de estrategia seleccionados de realizar una interacción con los investigadores. Desde el punto de vista cognitivo, todos dieron énfasis a la intelección. No obstante, el juego planteaba diferentes caminos interpretativos, vías distintas de posibilidad de jugada. Desde esa perspectiva encontramos en las tres ciudades el elemento común de privilegiar inteligibilizar la realidad por sobre la acción en sí misma. Luego, podemos decir que la recurrencia encontrada en todos los casos desde un punto de vista temático es la presencia de una hegemonía de lo intelectivo en función de la economía y de la implementación material en Antofagasta, mientras que en Temuco la mitad de los sujetos privilegian el desarrollo económico y en Santiago, la tendencia es la construcción de infraestructura material sólida. Cabe destacar que un sujeto por ciudad se preocupó de todos los elementos del juego.

Luego, el procesamiento cognitivo detectado está en correspondencia con un fractal productivo tecnológico complejo, sea a nivel de poder operar con una tecnología que haga conexionar espacios paralelos, o sea, que el sujeto tiene la competencia fractal de conexionar diferentes espacios de la vida social y articularlos en relaciones múltiples, predominando lo intelectivo, pues es de la intelección desde donde opera la acción.

La investigación detectó que hay una estrecha relación entre el procesamiento cognitivo y el procesamiento perceptivo, pues los sujetos desarrollaron una forma de mirar en correspondencia con esta búsqueda de procesamiento paralelo de la información. Esto es, la mirada focaliza en los casos en que el procesamiento no era narrativo (la mayoría) todo tipo de imágenes visuales. La percepción entonces no opera como estímulo-respuesta sino que lo que se percibe está en correspondencia a un esquema previo. La mirada establece un recorrido visual y lo hace a través de los ojos y/o del movimiento de la cabeza, y dichos recorridos son referidos a la pantalla, a la máquina misma (joistick o mouse, teclado) y eventualmente el contexto. Los movimientos tienden a ser: 1) múltiples, esto es no hay una sola figura que se repite, sino que el sujeto incorpora varios recorridos de mirada a la vez, lo que significa que el sujeto trata de aprehender el todo y no tiene un lugar privilegiado de la mirada; 2) tienden a ser azarísticos en el sentido de que lo que se repite es el esquema geométrico que de allí se configura, pero no una figura estable, lo que remite a esta necesidad de ligar elementos figurales de un espacio perceptivo con otro para de allí percibir una configuración global; 3) menor predominio de los recorridos verticales y horizontales en línea recta, pues el sujeto no solamente mira de arriba abajo, de izquierda a derecha, sino que quiere estar informado de todo lo que pasa; 4) presencia de paneos (recorridos curvos de la mirada), es decir, que registra todos los elementos del recorrido a diferencia de los movimientos rectos que perciben el punto de inicio y el final del movimiento, en un punto intermedio se encuentran las figuras quebradas (movimientos rectos que permiten captar tres o más espacios), lo que significa que a diferencia de la mirada horizontal-vertical, el recorrido curvo capta lo que está en los alrededores de la imagen no dejando fuera nada.

Esta competencia perceptiva le permite al sujeto operar con máquinas y tecnologías complejas y operar con espacios paralelos, pues percibe perfectamente un amplio espectro visual, sin una focalización narrativa de un solo espacio, como nuestro fractal combate realizaba, sino que adquiere la competencia del mirar tecnológico que le permite operar con

\footnotetext{
${ }^{23}$ VARELA, F. Op. Cit. Pág. 55.
} 
redes complejas. Castells analizando la brecha digital detecta que no se trata sólo de un acceso desigual, sino que ello tiene implicancias en la constitución de las competencias ligadas al saber y con ello la disminución de sus posibilidades de sobre montar sus debilidades económicas: "la educación, la información, la ciencia y la tecnología llegan a ser las fuentes cruciales de la creación de valor en la economía fundada sobre internet" 24 .

Esto es, Castells pone énfasis no sólo en cómo ha cambiado la economía global, sino en que operar en esta sociedad en redes supone habilidades cognitivas ligadas al uso de las nuevas tecnologías, donde vastos sectores del tercer mundo no tendrían acceso, y luego en ellas la "gran mayoría de la población no puede allí ser empleada puesto que no tiene las competencias requeridas" 25 , polarizándose el mercado de trabajo y la sociedad en forma dual. Esa dualidad, señalada por Castells, se deja ver en la antítesis de los fractales combate/estrategia, desde el punto de vista perceptivo y cognitivo.

\section{Brecha digital y estructuraciones cognitivas y perceptivas recurrentes en los usuarios de video-juegos de aventura gráfica (11-12 y 16-19 años)}

Los video-juegos de aventura gráfica, en la cual el jugador participa de ser un personaje de un filme son de complejidad media, situándose a medio camino de los dos precedentemente sintetizados. No podemos, en los límites espaciales del presente artículo, exponer con detalle los datos encontrados e inteligibilizados. Sólo diremos que en Santiago de los casos analizados, un 50\% entiende el juego, en Antofagasta el $17 \%$ de los jugadores, $\mathrm{y}$ en Temuco un $33 \%$.

La ausencia de percepción de los diferentes espacios y de las relaciones de articulación se debe en definitiva a un elemento invariante detectado en todos los usuarios de Santiago, Antofagasta y Temuco, que es focalizarce en el espacio narrativo de los personajes y en las secuencias de acción implicadas. hay un elemento presente en las tres ciudades que es clarificador: como todos buscan focalizar personajes y prever secuencias de acción no miran la totalidad. Sus formas estables de mirar recurrentes son legibles por la geometría euclidiana línea recta, horizontal-vertical, la inserción de rectas oblicuas, menor se detecta la presencia de curvas que en el fondo son la misma manifestación que las oblicuas con la diferencia que en teoría permiten percibir más, pero como los sujetos están interesados en percibir la acción y los personajes de la mirada curva no emerge un mayor ángulo de mirar. Luego, los usuarios de video-juegos de aventura gráfica tienden a percibir varios espacios, pero hacen de ello un tratamiento paralelo de la información de índole fundamentalmente narrativa, esto es, en función de una tarea productiva, una acción concreta que se pretende implementar por lo que retienen perceptiva y cognitivamente complejidades medias, sin percibir más que la tarea encomendada, por lo que es posible decir, respecto a la brecha digital, que se sitúan como mediadores entre el fractal combate y el fractal estrategia y los casos analizados avalan esto, pues no hay un comportamiento homogéneo respecto a la identificación de espacios, pero sí respecto a focalizar como objeto de la mirada la tarea pedida, la acción, lo narrativo.

\section{Brecha digital y estructuraciones cognitivas y perceptivas enactivas}

Del estudio, también se detecta que las categorías cognitivas y perceptivas son históricas, enactivas. Esto es, no son invariantes, sino que susceptibles de modificarse por

\footnotetext{
${ }^{24}$ CASTELLS, M. "La galaxie internet”. Pág. 322. Ed. Fayard. París, 2002.

${ }^{25}$ CASTELLS, M. Op. Cit. Pág. 322.
} 
la historia. Significativo es el caso del segmento etario 5-6 años, donde todavía no existe una adscripción a ser un usuario de un tipo de video-juego y donde, si bien es cierto que la mayoría no entendió los juegos, el problema cognitivo para ellos, era entender la máquina, y desarrollaron tratamientos de espacios paralelos, y percibieron focalizando toda la máquina, la pantalla y los investigadores, salvo los casos muy marcados por una perspectiva catártica minoritaria que se expresaba muy bien en los dibujos que elaboraron. 